\title{
Smartphone-Based Paper Microfluidic Particulometry of Norovirus from Environmental Water Samples at the Single Copy Level
}

\author{
Soo Chung, ${ }^{\dagger}$ Lane E. Breshears, ${ }^{\ddagger}$ Sean Perea, ${ }^{\S}$ Christina M. Morrison, "Walter Q. Betancourt," \\ Kelly A. Reynolds, ${ }^{\perp}$ and Jeong-Yeol Yoon* ${ }^{\dagger}+{ }^{\dagger}$
}

${ }^{\dagger}$ Department of Biosystems Engineering, ${ }^{\ddagger}$ Department of Biomedical Engineering, ${ }^{\S}$ Department of Chemical and Environmental Engineering, "Department of Soil, Water and Environmental Science, and ${ }^{\perp} \mathrm{Mel}$ and Enid Zuckerman College of Public Health, The University of Arizona, Tucson, Arizona 85721, United States

Supporting Information

\begin{abstract}
Human enteric viruses can be highly infectious and thus capable of causing disease upon ingestion of low doses ranging from $10^{0}$ to $10^{2}$ virions. Norovirus is a good example with a minimum infectious dose as low as a few tens of virions, that is, below femtogram scale. Norovirus detection from commonly implicated environmental matrices (water and food) involves complicated concentration of viruses and/or amplification of the norovirus genome, thus rendering detection approaches not feasible for field applications. In this work, norovirus detection was performed on a microfluidic paper analytic device without using any sample concentration or nucleic acid amplification steps by directly imaging and counting on-paper aggregation of antibody-conjugated, fluorescent submicron particles. An in-house developed smartphone-based fluorescence microscope and an image-processing algorithm isolated the particles aggregated by antibody-antigen binding, leading to an extremely low limit of norovirus detection, as low as 1 genome copy $/ \mu \mathrm{L}$ in deionized water and 10 genome copies $/ \mu \mathrm{L}$ in reclaimed wastewater.
\end{abstract}

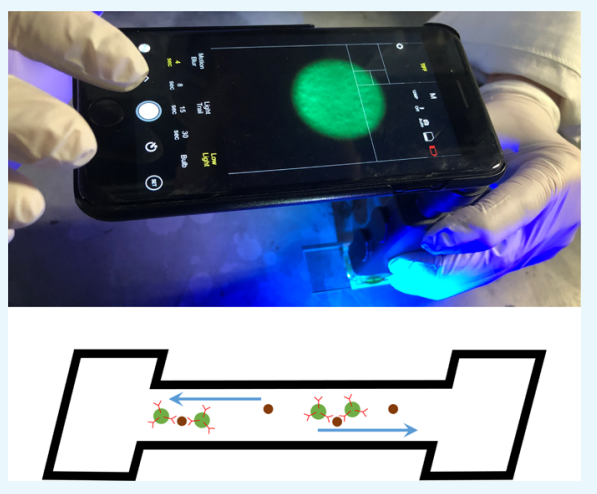

\section{INTRODUCTION}

Human enteric viruses are small infectious agents that can cause gastrointestinal disease upon ingestion of very low doses. Detection of these viruses requires an extremely low limit of detection (LOD), especially when assessing viruses in reclaimed wastewater or unconfined aquifers used as sources of drinking water. Norovirus is one of such well-known examples and is the most common cause of epidemic and sporadic gastroenteritis worldwide. ${ }^{1}$ Studies have indicated that norovirus infection can occur upon exposure to as few as 18 virions. ${ }^{2,3}$ Highly sensitive detection methods are needed for assessing exposure to norovirus, especially considering that the methods for virus recovery and concentration from environmental matrices are rather inefficient. In addition, the infectivity of human noroviruses by in vitro cell culture has proven to be quite complex (only possible in stem cell-derived human enteroids), ${ }^{4}$ which prevents the use of traditional culture-based assays for evaluating virus infectivity in environmental matrices. Because of this limitation, norovirus has been assayed by either reverse transcription polymerase chain reaction $(\mathrm{RT}-\mathrm{PCR})^{5}$ or sandwich immunoassay ${ }^{6}$ techniques. While RT-PCR-based techniques do provide necessary specificity for detection and identification of norovirus, these molecular methods are susceptible to inhibition by multiple components associated with environmental matrices and fail to provide sufficient rapidity and field-applicability. ${ }^{7}$ Immunoassay techniques are simpler than RT-PCR and have the potential to be incorporated on a microfluidic platform. Specifically, microfluidic paper analytic devices ( $\mu$ PADs) have shown numerous advantages over silicone-based microfluidic devices, as they are lightweight, easy to fabricate via wax printing (no lithography), use spontaneous flow by capillary action, and have potential on-chip filtration capability. However, optical detection of low concentrations of pathogens has rarely been demonstrated on paper substrates because paper is optically opaque and non-homogeneous (porous), generating substantial background scatter and reflection. So far, single virus copy level detection of norovirus has rarely been demonstrated on paper substrates (including lateral flow assays and $\mu$ PADs). While single copy level detection of other virus targets has indeed been demonstrated on paper substrates $(20$ copies of Ebola, 20 copies $/ \mu \mathrm{L}$ of pseudorabies, and 1 copy $/ \mu \mathrm{L}$ of HIV), all of them required nucleic acid amplifications, most notably isothermal methods such as loop-mediated isothermal amplification (LAMP). ${ }^{10-12}$ Such methods are not sufficiently simple for field-based applications (requiring a heater and thermostat system plus an expensive isothermal amplification kit) and cannot be considered near-real-time (just the amplification part can take from $15 \mathrm{~min}$ to $2.5 \mathrm{~h}$ ). As described previously, immunoassay on $\mu \mathrm{PAD}$ without sample

Received: March 20, 2019

Accepted: June 14, 2019

Published: June 27, 2019 
(a)

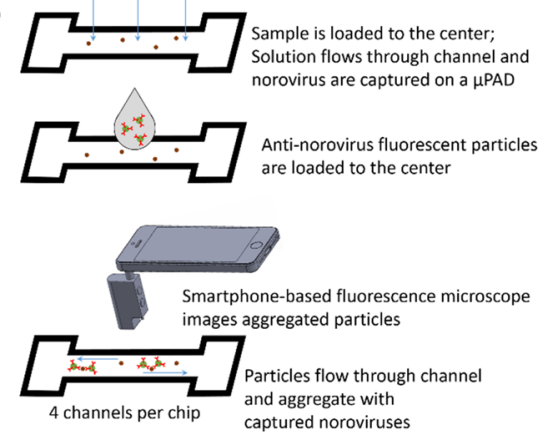

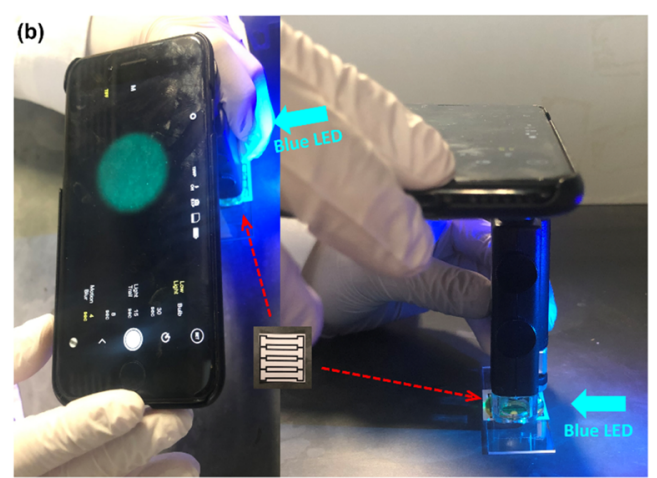

Figure 1. Schematic illustration of norovirus assay on $\mu \mathrm{PAD}$ using a smartphone-based fluorescence microscope. (a) Norovirus solutions (5 $\mu \mathrm{L}$ ) are added directly to the main channel of $\mu \mathrm{PAD}$ (made out of nitrocellulose), followed by $2 \mu \mathrm{L}$ of anti-norovirus particle suspension ( $0.001 \% \mathrm{w} / \mathrm{v}$ ). Solutions spread throughout the entire channel by capillary action, which are imaged by a smartphone-based fluorescence microscope. (b) Blue LED $(480 \mathrm{~nm})$ irradiates the $\mu \mathrm{PAD}$ from the side. A smartphone with a microscope attachment and a bandpass filter $(525 \pm 20 \mathrm{~nm}$; green emission) captures the fluorescent images of a $\mu \mathrm{PAD}$ (photograph courtesy: Soo Chung and Sean Perea; copyright 2019).

concentration and/or nucleic acid amplification is the ideal method for field-based norovirus detection, which has unfortunately not been demonstrated at the single virus copy level. The LODs of paper-based norovirus immunoassays ranged from $10^{4}$ to $10^{6}$ copies $/ \mu \mathrm{L}(=10 \mathrm{fg} / \mu \mathrm{L}$ to $1 \mathrm{pg} / \mu \mathrm{L}$, as the weight of a single norovirus particle is approximately $10 \mathrm{ag}$ considering its diameter of $35-40 \mathrm{~nm})^{13}$ without concentration or amplification ${ }^{14,15}$ and $10^{2}$ copies $/ \mu \mathrm{L}$ with $1 \mathrm{~h}$ reaction of signal amplification. ${ }^{16}$

In this work, we attempted to "visualize" the norovirusinduced particle immunoagglutination down to the single virus copy level directly on a $\mu \mathrm{PAD}$ toward field-based applications. Antibody-conjugated, submicron, fluorescent polystyrene particles were used on $\mu \mathrm{PAD}$ to quantify norovirus. The $\mu \mathrm{PAD}$ allows the antibody-conjugated particles and norovirus to "flow" through paper pores spontaneously via capillary action, which is much faster and more effective than passive, diffusional mixing. As the submicron particles move much slower than norovirus, unbound noroviruses can also be washed from the antibody-conjugated particles, potentially eliminating a separate washing step. ${ }^{17}$ The extent of particle aggregation caused by antibody-antigen binding was correlated to the norovirus concentration in the samples. A smartphone-based fluorescence microscope was used to identify and quantify these aggregated particles to provide additional field applicability. Only the aggregated particles could be isolated through image processing, enabling extremely sensitive detection down to the single virus copy level. Neither sample concentration nor nucleic acid amplification steps are necessary due to such an extremely low LOD. This novel method is wholly different from other optical biosensing methods where their signals are ensemble-averaged, that is, specific, nonspecific, and background signals are not fully isolated. By securing direct evidence of particle aggregation, credibility and accuracy of the assay could be improved. In addition, it is also entirely different from other imaging-based virus counting methods, where host cells are infected with target viruses. ${ }^{18}$ Such methods require in vitro cultivation of noroviruses, which is costly and time-intensive, ${ }^{19}$ and most importantly, are complex and difficult for norovirus.

To accomplish our goal, we designed and tested a smartphone-based fluorescence microscope to image aggregated particles directly on a wax-printed $\mu \mathrm{PAD}$ (Figure 1a). In this novel method, norovirus target solutions ( $5 \mu \mathrm{L}$ each) were first loaded on $\mu$ PADs, followed by the addition of antibodyconjugated, yellow-green fluorescent polystyrene particle suspension that resulted in particle aggregation (i.e., immunoagglutination). This alternative approach enabled the antibody-conjugated particles to spread and flow through the entire channel, allowing them to be imaged separately and minimizing nonspecific aggregation. In addition, much lower concentration of the antibody-conjugated particles (0.001$0.002 \%$ ) was used for the particle suspension than those used in other particle immunoassays, which also contributed to minimizing nonspecific aggregation. A smartphone-based fluorescence microscope (Figure 1b) was constructed to fluorescently image the several different areas of a $\mu \mathrm{PAD}$ channel. Through a novel image processing algorithm, only the aggregated particles were isolated to relate them to the norovirus concentration. Field water samples-tap water and reclaimed wastewater-were also evaluated.

The overarching aim of this work is to demonstrate extremely low LOD, preferably near to the single virus copy level (corresponding to $\sim 10 \mathrm{ag}$ ), in a rapid and field-ready manner using a $\mu \mathrm{PAD}$ and smartphone-based fluorescence microscopy.

\section{RESULTS AND DISCUSSION}

Benchtop Microscope Assays. Initially, $\mu \mathrm{PAD}$ assays were conducted for assessing the norovirus capsids using a benchtop fluorescence microscope and subsequent ImageJ analysis. All serial dilutions were made in $1 \mathrm{~mL}$ volume and vortex-mixed to ensure that there were sufficient amounts of norovirus in each dilution even at the lowest concentration. For each assay, four different areas of a single channel were imaged. Through size analysis, the locations of fluorescent particles (both nonaggregated and aggregated) could easily be determined, which showed the pixel intensities of at least 100 (out of 255). Distinction could also be made between nonaggregated and aggregated particles using the pixel area of 50. Therefore, the raw images were processed to eliminate the pixels with $<100$ intensity (to remove background) and the pixel area $<50$ (to remove nonaggregated particles). From these four processed images from a single $\mu \mathrm{PAD}$ channel, a number of pixels were added together to yield a single data point. This number corresponded to the extent of particle aggregation and thus norovirus concentration. Experiments were repeated 3-4 times, each time using a different $\mu \mathrm{PAD}$. 

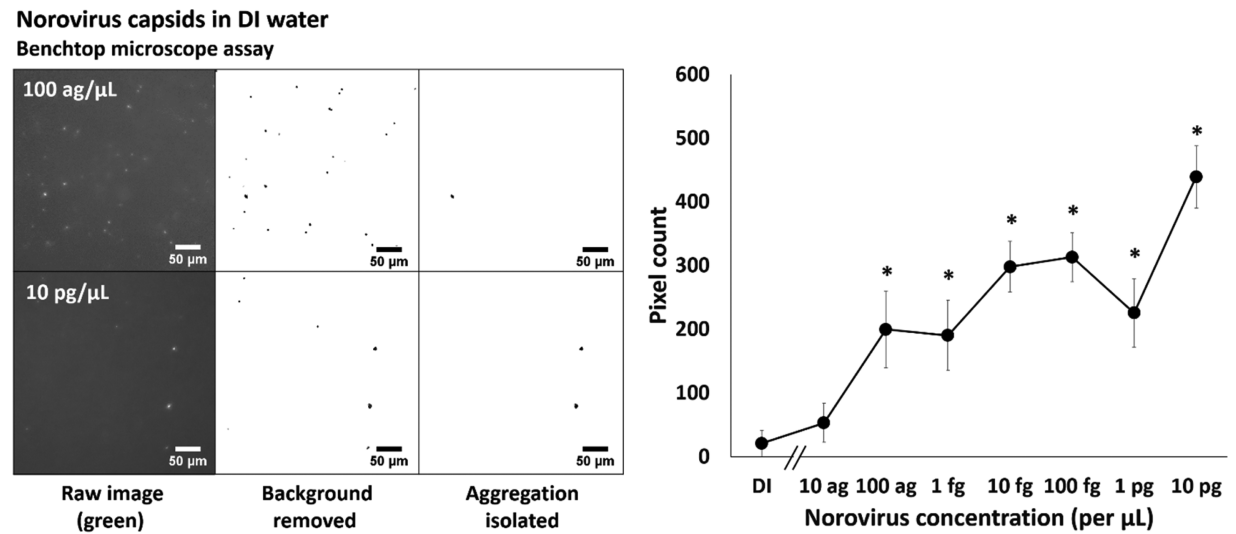

Figure 2. Benchtop microscope assay results for norovirus capsids. For each assay, four different areas of a single channel were imaged and analyzed to obtain the pixel counts of aggregated particles. The pixel counts from 4 different images were added together to yield a single data point. Only green channel images were used. Experiments were repeated three times $(0-1 \mathrm{fg} / \mu \mathrm{L})$ or 4 times $(10 \mathrm{fg} / \mu \mathrm{L}$ to $10 \mathrm{pg} / \mu \mathrm{L})$, each time using a different $\mu \mathrm{PAD}$. Error bars represent standard errors of such 3-4 assays. * indicates statistically significant difference $(p<0.05$ with Wilcoxon rank sum test) from a negative control sample. Left: representative raw, background-removed, and nonaggregated particles-removed images (captured by a benchtop fluorescence microscope and processed with ImageJ) of a $\mu \mathrm{PAD}$ at given norovirus capsid concentrations. These images are zoomed-in versions $(400 \mu \mathrm{m} \times 400 \mu \mathrm{m})$ to clearly show the particles; the actual images used in the assays are $1.060 \mathrm{~mm}$ wide and $0.792 \mathrm{~mm}$ long. Right: average pixel counts from $\mu \mathrm{PAD}$ are plotted against norovirus capsid concentrations, using a benchtop fluorescence microscope and Image processing.
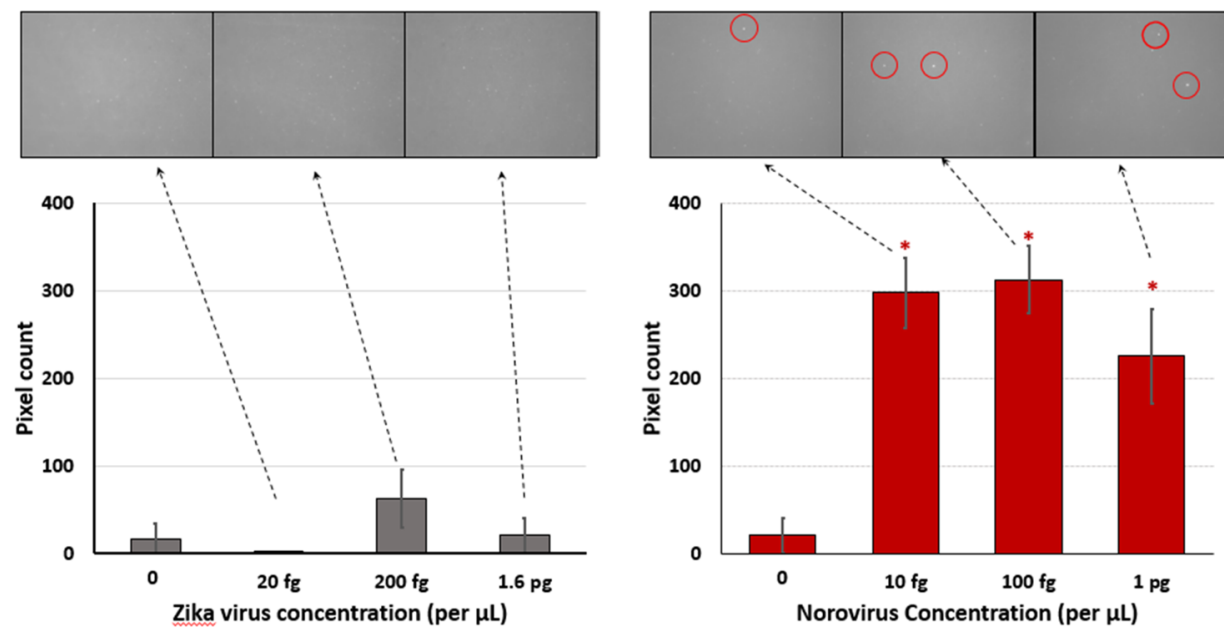

Figure 3. Specificity test. Three different concentrations of Zika virus and norovirus were tested with anti-norovirus-conjugated particles. Benchtop microscope assays and ImageJ analyses were used. Other experimental conditions are identical to those shown in Figure 2.

Representative zoomed-in images (raw and processed) are provided in Figure 2 to the left to better represent the aggregated particles. To confirm whether the pixel area truly represented the particle size and distinguished the aggregated from non-aggregated particles, fluorescence and light microscopic images were obtained for the aggregated particles on a $\mu \mathrm{PAD}$ and processed in the same manner (Figure $\mathrm{S} 1$ ). Two different types of particles were observed in fluorescence images, where the smaller ones potentially represent the nonaggregated particles and the bigger ones the aggregated particles. Note that the particle size $(0.5 \mu \mathrm{m})$ is comparable to the emission wavelength $(525 \mathrm{~nm})$ of fluorescent particles. With light microscopic images, however, only the bigger particles could be observed, exactly at the same locations of bigger sized particles in the fluorescence microscopic images. As the particle size $(0.5 \mu \mathrm{m})$ is smaller than the upper limit of visible wavelength $(400-750 \mathrm{~nm})$, it will be difficult to image the $0.5 \mu \mathrm{m}$, nonaggregated particles, while the aggregated particles $(>0.8 \mu \mathrm{m})$ can be imaged relatively easily.
The averages and standard errors of these pixel counts from 3 to 4 independent assays were plotted against the norovirus concentration in Figure 2 to the right. As the sample size is relatively small, it was difficult to assume normal distribution for each data point. Therefore, the nonparametric Wilcoxon rank sum test was conducted for each data point in comparison to the zero-concentration data point [in deionized (DI) water] as a negative control. The lowest concentration of norovirus capsid that passed the Wilcoxon rank sum test $(p<0.05)$ was $100 \mathrm{ag} / \mu \mathrm{L}$, which is the LOD of this assay. All concentrations from $100 \mathrm{ag} / \mu \mathrm{L}$ to $10 \mathrm{pg} / \mu \mathrm{L}$ were also significantly different from the zero concentration (negative control), indicating that the particle aggregation was highly correlated to the norovirus presence and minimum nonspecific aggregation. This LOD is several orders of magnitude lower than $0.25-12.5 \mathrm{pg} / \mu \mathrm{L}$ $(=\mathrm{ng} / \mathrm{mL}$ ) with the commercial lateral flow assays (including immunoCatch-Noro from Eiken Chemical, GE test Noro Nissui from Nissui Pharmaceutical, and Quick Navi-Noro 2 from Denka Seiken) and $10-100 \mathrm{fg} / \mu \mathrm{L}$ as reported in the 

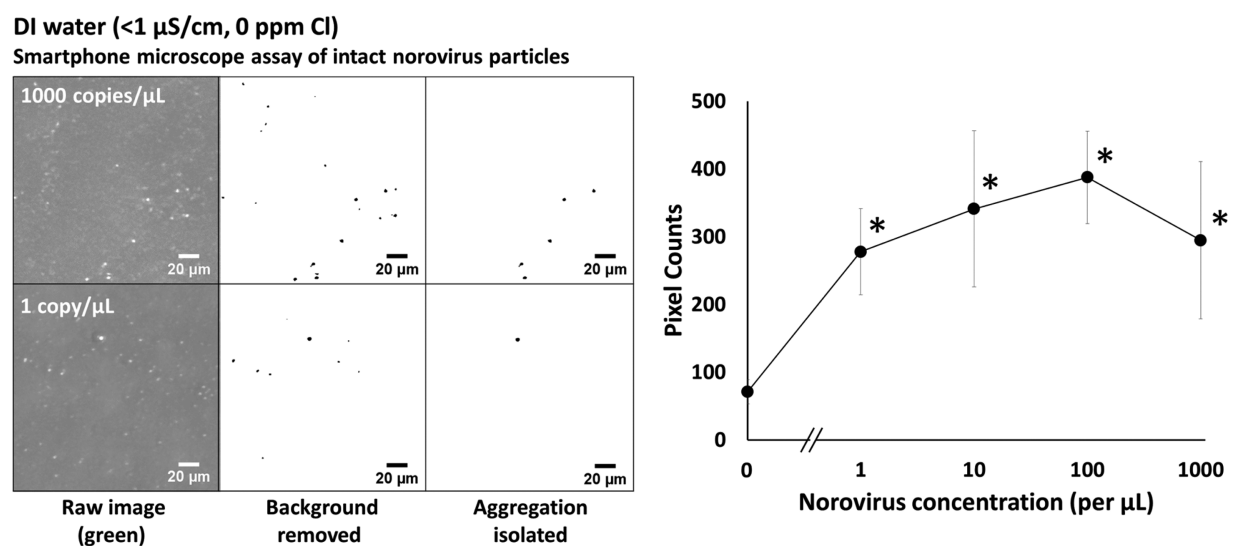

Figure 4. Smartphone assay results for intact norovirus in DI water. For each assay, four different areas of a single channel were imaged and analyzed to obtain the pixel counts of aggregated particles. The pixel counts from four different images were added together to yield a single data point. Both green and red channels were combined to maximize pixel intensities. Experiments were repeated three times, each time using a different $\mu \mathrm{PAD}$. Error bars represent standard errors of such three assays. The Wilcoxon rank sum test was performed and $*$ indicates statistically significant difference $(p<0.05)$ from a negative control sample. Left: representative raw and processed images of $\mu$ PAD at given intact norovirus concentrations. These images are zoomed-in versions $(196 \mu \mathrm{m} \times 196 \mu \mathrm{m})$ to clearly show the particles. Right: Average pixel counts from $\mu$ PAD are plotted against intact norovirus concentrations using a smartphone-based fluorescent microscope and a MATLAB code.

recent literature utilizing nanostructures as well as laboratory equipment such as a microplate reader ${ }^{24}$ or surface plasmon resonance equipment. ${ }^{25}$ Because the weight of a single norovirus particle is approximately 10 ag considering its diameter $(35-40 \mathrm{~nm}),{ }^{13}$ this LOD value is close to a single virus particle level within an order of magnitude.

Specificity Test. To evaluate the specificity of this assay, Zika virus was assayed using anti-norovirus-conjugated particles and compared with the results of norovirus assay. Experimental conditions were identical to those of norovirus assays. As shown in Figure 3, the pixel counts were much smaller with Zika virus than with norovirus. All Zika virus concentrations were not significantly different from the zero concentration (negative control) using the nonparametric Wilcoxon rank sum test. Taking these results together, satisfactory specificity was achieved by the assay at least for the given experimental conditions.

Smartphone Microscope Assays. Next, the same experiments were repeated while replacing norovirus capsids with intact noroviruses (refer to the Methods section for the preparation of intact norovirus and RT-qPCR assay). The $\mu \mathrm{PAD}$ assays were conducted for assessing intact norovirus using a smartphone microscope shown in Figure $1 \mathrm{~b}$ and MATLAB mobile graphical user interface (GUI) app (Figure S2). Intact noroviruses were initially diluted in DI water. Again, all serial dilutions were made in $1 \mathrm{~mL}$ volume and vortex-mixed to ensure that there were sufficient amounts of norovirus even at the lowest concentration (1000 genome copies in 1 copy $/ \mu \mathrm{L}$ sample). Because the smartphone constantly attempts to compensate for lighting bias and exposure, and to adjust white balance, the overall brightness of raw images was different from assay to assay. Therefore, the raw images (already square-cropped circumscribing circular field of view) were processed to eliminate the pixels with the intensities smaller than the overall mean +50 (out of 255; to remove background noise), binarized, and further processed to eliminate the pixel areas smaller than 30 (to remove nonaggregated particles). Refer to the Methods section for details. Similar to the benchtop microscope assays, four different areas of a single channel were imaged and analyzed, and the pixel counts were added together to yield a single data point. Experiments are repeated three times, each time using a different $\mu \mathrm{PAD}$. The results are depicted in Figure 4, showing the representative, zoomed-in images (raw, background removed, and aggregation isolated) for $1 \mathrm{copy} / \mu \mathrm{L}$ (the lowest concentration assayed) and 1000 copies $/ \mu \mathrm{L}$ (the highest concentration significantly different from the negative control, i.e., virus-free deionized water) to the left, and the plot of average pixel counts against the norovirus concentration (genome copies per $\mu \mathrm{L}$ ) to the right. All final processed images without zoom-in are summarized in Figure S3. The lowest concentration that is significantly different $(p<0.05$ with Wilcoxon rank sum test) from the control (virus-free DI water) is 1 copy $/ \mu \mathrm{L}$, the LOD of this assay. It corresponds to $10 \mathrm{ag} / \mu \mathrm{L}$ considering the size of a norovirus particle, $35-40$ $\mathrm{nm},{ }^{13}$ and is 1 order of magnitude lower than that of assaying norovirus capsids, $100 \mathrm{ag} / \mu \mathrm{L}$. This can be attributed to the fact that the norovirus capsids were recombinant proteins that might have inferior affinity to the anti-norovirus compared to the intact norovirus samples. Concentrations of 10 and 100 copies $/ \mu \mathrm{L}$ are also significantly different from the control $(p<$ $0.05)$. The average pixel count at the highest concentration, 1000 copies $/ \mu \mathrm{L}$, is slightly smaller than that of 100 copies $/ \mu \mathrm{L}$, indicating that this concentration is outside the linear range of assay. In other words, there were too many virus particles that "consumed" all antibodies, which subsequently failed to connect antibody-conjugated particles together. Despite this, it is still substantially higher than the negative control $(p<$ $0.05)$.

To further confirm this extremely low LOD of 1 copy $/ \mu \mathrm{L}$, the number of aggregated particle clusters (not the pixel counts) in four different images (from a single $\mu \mathrm{PAD}$ channel) was totaled together. The total average from the three different assays was $6 \pm 1$. The volume of the loaded sample of $5 \mu \mathrm{L}$, corresponding to 1 copy $/ \mu \mathrm{L} \times 5 \mu \mathrm{L}=5$ copies, is comparable to the above count of particle clusters. It should be noted that a portion of such clusters may not represent "true" aggregation caused by antibody-antigen binding but rather nonspecific aggregation. The result shown in Figure 4 further corroborate this fact, as the pixel counts with zero concentration is $\sim 80$, representing a small extent of nonspecific aggregation, while those with $1 \mathrm{copy} / \mu \mathrm{L}$ is $\sim 280$. In addition, the genome copy 

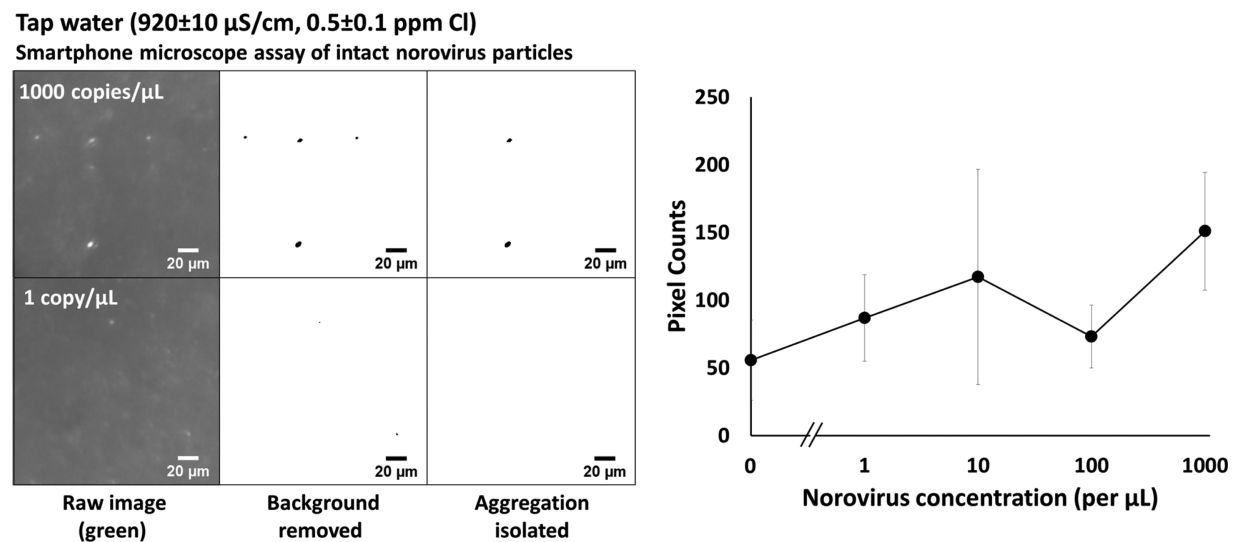

Figure 5. Smartphone assay results for tap water. Other experimental conditions are identical to those shown in Figure 3, except that the assays were repeated six times.
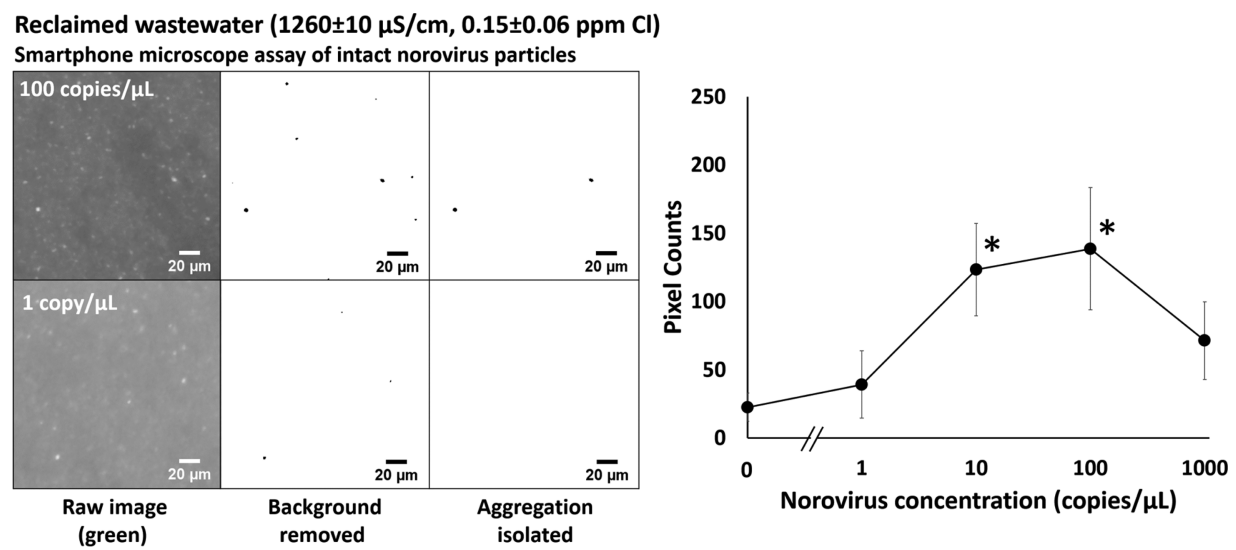

Figure 6. Smartphone assay results for reclaimed wastewater. Other experimental conditions are identical to those shown in Figure 3, except that the assays were repeated six times.

number (evaluated by RT-qPCR) does not truly represent the number of "all" virus particles, which can be higher. It is also possible that the sample contained free antigens and fragments in addition to intact viruses, which could also enable particle immunoagglutination.

Smartphone Microscope Assays with Field Water Samples. We then proceeded to further evaluate this method for two different field water samples: intact noroviruses were spiked into tap water and reclaimed wastewater. Water samples were serially diluted using the same tap water or reclaimed wastewater; thus the sample matrices were undiluted. As described in the Methods section, the raw images were processed to remove background noise using the cut-off intensities of the overall mean $+40,+45$, or +50 . These images were then binarized and further processed to remove nonaggregated particles (isolating only the aggregated particles) using the cut-off pixel areas of 30. The cut-off intensities (mean $+40,+45$, or +50 ) were selected that minimized the presence of background noise, represented by single pixels not clustered together. Particles were always represented by clusters of pixels. Experiments were repeated 6 times with both tap water and reclaimed wastewater, each time using a different $\mu \mathrm{PAD}$.

The assay results with tap water are depicted in Figure 5. No data points passed the Wilcoxon rank sum test $(p>0.05)$, while the $p$ value was the smallest $(0.063)$ with the highest concentration of 1000 copies $/ \mu \mathrm{L}$. While the overall pixel counts generally increased from the negative control, they were not significantly different. Additionally, the pixel counts are also lower (80-160) than those with DI water (270-390). These results can be attributed to electrolytes common in tap water (its conductivity was $920 \pm 10 \mu \mathrm{S} / \mathrm{cm}$ ) or its high chlorine content $(0.5 \pm 0.1 \mathrm{ppm})$.

Identical experiments were repeated with reclaimed wastewater. The assay results with reclaimed wastewater are shown in Figure 6. While the pixel counts $(40-140)$ are still lower than those with DI water (270-390) and comparable to those of tap water (40-140), the lowest concentration that was significantly different (with Wilcoxon rank sum test) from the negative control (unspiked reclaimed wastewater) is 10 copies $/ \mu \mathrm{L}$ (corresponding to $100 \mathrm{ag} / \mu \mathrm{L}$ ), again close to the single virus copy level. The overall curve also resembles the one with DI water, that is, an increase up to 100 copies $/ \mu \mathrm{L}$ followed by a decrease at 1000 copies $/ \mu \mathrm{L}$. The conductivity of reclaimed wastewater was $1260 \pm 10 \mu \mathrm{S} / \mathrm{cm}$, which was even higher than that $920 \pm 10 \mu \mathrm{S} / \mathrm{cm}$ of tap water, while its chlorine content was $0.15 \pm 0.06 \mathrm{ppm}$, significantly lower than that $0.5 \pm 0.1 \mathrm{ppm}$ of tap water. To confirm the effect of chlorine to our assay, a control experiment was performed by adding 0.5 and $5 \mathrm{ppm}$ chlorine to DI water, and the results are shown in Figure S4. Compared to the DI water results (Figure 4 ), the error bars were larger and comparable to those with the tap water results (Figure 5). With $0.5 \mathrm{ppm}$ chlorine, a very narrow linear response up to 10 copies $/ \mu \mathrm{L}$ was observed 
followed by premature saturation. Such narrow linearity could not be found with $5 \mathrm{ppm}$ chlorine, 1 order of magnitude higher concentration than that of tap water. Thus, chlorine could be responsible for rendering the assay results less reproducible, although the role of electrolytes in tap water could not be ruled out entirely. In addition, chlorine might have adversely affected the availability of antibody-conjugated particles. (Chlorines can easily be removed by simply letting them to evaporate from water samples).

The excellent LODs in DI water and reclaimed wastewater can be attributed to many factors. Most importantly, we developed an image-processing algorithm that isolated only the immunoagglutinated particles and counted the total number of such pixels. While a large number of fluorescent dyes and/or nanoparticles were necessary to collect sufficiently strong signals in other optical detection methods, only a small number of particles were necessary for individual counting. It also contributed to minimizing nonspecific aggregation and facilitating capillary action-driven washing. In addition, most immunoagglutinated particles were retained and quantified in the field of view through direct imaging and counting on a paper substrate, enabling single virus copy level detection.

\section{CONCLUSION}

To summarize, we demonstrated an easy-to-use, low-cost, and extremely sensitive assay for detecting waterborne virus pathogens that does not require concentration, in vitro cell culture, and/or nucleic acid amplification. $\mathrm{A} \mu \mathrm{PAD}$ was fabricated via wax printing, and noroviruses were captured directly on a $\mu \mathrm{PAD}$. Antibody-conjugated submicron particles were then loaded to a $\mu \mathrm{PAD}$ and resulting particle aggregation was imaged directly on a $\mu \mathrm{PAD}$ surface. An image analysis algorithm was developed to isolate only the aggregated particles while removing the background, generating visually convincing assay results that were not affected by lighting biases and perturbations. Benchtop fluorescence microscope and subsequent ImageJ analysis were initially performed to identify and quantify norovirus capsids in DI water. Smartphone-based fluorescence microscope and original MATLAB mobile GUI app were then used to quantify intact norovirus samples in various field water samples. The LODs with smartphone assays were $1 \mathrm{copy} / \mu \mathrm{L}$ in DI water and 10 copies $/ \mu \mathrm{L}$ in reclaimed wastewater at single virus particle level. Because of these extremely low LODs, virus concentration or nucleic acid amplification steps were not necessary. The results with tap water were inferior, presumably because of its high chlorine content. This can be easily resolved by simply letting chlorine to evaporate from water samples. Additionally, a separate "control" channel is not necessary, which is typically required for other optical microfluidic biosensing. This method with extremely low LOD can also be applied for detection of any other viral pathogens in environmental samples such as food, water, and fomites.

\section{METHODS}

$\mu$ PAD Fabrication. A ColorQube wax printer (Xerox Corporation; Norwalk, CT, USA) was used to print the microfluidic design (Figure 1a) onto a nitrocellulose paper (Hi-Flow Plus Membrane, catalog number HF07502XSS; Millipore; Billerica, MA, USA). Each chip has four wax-printed channels (21 mm long and $2.4 \mathrm{~mm}$ wide). Each chip was heated on a hot plate (Corning; Corning, NY, USA) at $120^{\circ} \mathrm{C}$ until the surface-printed wax was melted to fill the paper pores underneath.

Antibody Conjugation to Fluorescent Particles. The rabbit polyclonal antibody to norovirus capsid protein VP1 (anti-norovirus, catalog number ab92976; Abcam, Inc.; Cambridge, MA, USA) was used for assaying both norovirus capsids and intact noroviruses. Anti-norovirus was covalently conjugated to carboxylated, yellow-green fluorescent, polystyrene particles (particle diameter $=0.5 \mu \mathrm{m}$; Magsphere, Inc.; Pasadena, CA, USA). The fluorescent characteristics of these particles were reported by the manufacturer: maximum excitation at $480 \mathrm{~nm}$ (blue) and maximum emission at 525 $\mathrm{nm}$ (green). Prior to antibody conjugation, particles were prewashed with DI water to remove surfactants from the stock solution, through centrifuging at $9.9 \mathrm{~g}$ for $13 \mathrm{~min}$. The antibody was then conjugated to these fluorescent particles following a protocol described in detail elsewhere. ${ }^{20}$

Norovirus Sample Preparation. Initially, recombinant norovirus group-1 capsid (MyBioSource, Inc.; San Diego, CA, USA) was used as a target. Norovirus capsids were serially diluted in DI water from the $1 \mathrm{ng} / \mu \mathrm{L}$ stock solution to make $10 \mathrm{pg} / \mu \mathrm{L}, 1 \mathrm{pg} / \mu \mathrm{L}, 100 \mathrm{fg} / \mu \mathrm{L}, 10 \mathrm{fg} / \mu \mathrm{L}, 1 \mathrm{fg} / \mu \mathrm{L}, 100 \mathrm{ag} / \mu \mathrm{L}$, $10 \mathrm{ag} / \mu \mathrm{L}$, and $1 \mathrm{ag} / \mu \mathrm{L}$, all in $1 \mathrm{~mL}$ volume at $1: 10$ dilution each (4-10 serial dilutions). The systematic errors of pipettes were $\pm 0.8 \%$ for a $1000 \mu \mathrm{L}$ pipette and $\pm 0.6 \%$ for a $100 \mu \mathrm{L}$ pipette, resulting in the propagated errors of $2.0-3.1 \%$ for the given range of dilutions. These errors were too small to be represented as the horizonal error bars in the logarithmic scale $x$-axes in all plots.

Intact norovirus samples were collected from toilet fecal samples during an active norovirus outbreak. These samples were confirmed and quantified by quantitative reverse transcription polymerase chain reaction (RT-qPCR). Fecal samples were suspended in sterile phosphate buffered saline solution $(\mathrm{pH} 7.4)$ at $10 \% \mathrm{w} / \mathrm{v}$. These fecal suspensions were centrifuged at $1455 \mathrm{~g}$ for $10 \mathrm{~min}$ using Centriprep centrifugal filters (50 kDa cutoff; EMD Millipore, Burlington, MA, USA) to purify virus particles. The retentates $(\sim 0.75 \mathrm{~mL})$ were divided into aliquots of $200 \mu \mathrm{L}$ and frozen or subjected to nucleic acid extraction. To confirm and quantify norovirus, virus nucleic acids were extracted using the QIAmp viral RNA extraction kit (Qiagen, Chatsworth, CA, USA) and RT-qPCR assays were performed for three different genogroups of norovirus (GI, GII, and GIV) following previously reported assays. $^{21-23}$ GII norovirus RNA was predominantly detected from the fecal suspensions, with a viral load of approximately $10^{7}$ virus targets per $\mathrm{mL}$ of a stool supernatant. These fecal suspensions were serially diluted in various water samples (described in the following section) from the 10000 genome copies $/ \mu \mathrm{L}$ to obtain 1000 genome copies $/ \mu \mathrm{L}, 100$ copies $/ \mu \mathrm{L}$, 10 copies $/ \mu \mathrm{L}$, and $1 \mathrm{copy} / \mu \mathrm{L}$, again all in $1 \mathrm{~mL}$ volume at $1: 10$ dilution each ( $1-4$ serial dilutions). Using the same systematic errors of pipettes, the propagated errors were $1.0-2.0 \%$ for the given range of dilutions. Again, these errors were too small to be represented as the horizonal error bars in the logarithmic scale $x$-axes in all plots.

Specificity Test. Zika virus (attenuated virus particles; NATtrol Zika Virus Range Verification Panel; ZeptoMetrix Corporation, Buffalo, NY, USA) was used to evaluate the cross-reactivity of anti-norovirus with this assay. Both norovirus and Zika virus are single-stranded RNA viruses, have globular shapes, and are similar in size. Identical experiments were performed by substituting norovirus samples 


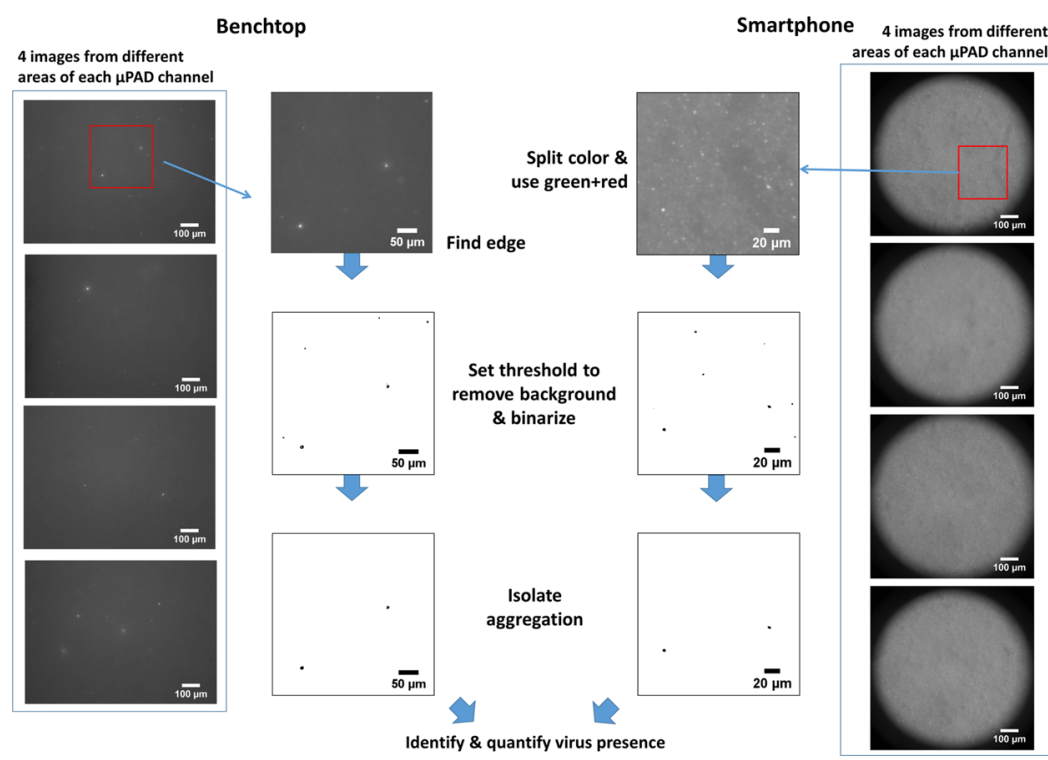

Figure 7. Image-processing algorithm using ImageJ for benchtop fluorescence microscopic images (left) and MATLAB GUI code for smartphone fluorescence microscopic images (right). Using the predetermined cut-off pixel intensity (to remove background) and pixel area (to isolate aggregated particles), along with binarization, a processed image is generated showing only the aggregated particles. The total pixel counts are added altogether from four different images from a single $\mu \mathrm{PAD}$ channel, which makes up a single data point. This experiment is repeated 3-6 times, each time using a different $\mu \mathrm{PAD}$, to evaluate the average pixel counts. Images in the first and last columns are raw images; those in the second and third columns are zoomed-in versions to clearly show the particles.

with Zika virus samples. The concentrations of Zika virus samples were $1.6 \mathrm{pg} / \mu \mathrm{L}, 200 \mathrm{fg} / \mu \mathrm{L}$, and $20 \mathrm{fg} / \mu \mathrm{L}$.

Water Samples. Various types of environmental water samples, spiked with known concentrations of norovirus, were tested in this work: DI water, drinking tap water, and reclaimed wastewater. The latter was produced in a facility utilizing primary sedimentation dissolved air flotation, four parallel fivestage Bardenpho processes, disk filtration, and chlorination. These water samples were tested for $\mathrm{pH}$, conductivity, and chlorine residual. $\mathrm{pH}$ was measured using the $\mathrm{pH}$ electrode and $\mathrm{pH}$ monitor (Pinpoint American Marine Inc.; Ridgefield, CT, USA). Conductivity was measured using the Ultrapen PT1 (Myron L Company; Carlsbad, CA, USA). Free chlorine residual was assayed by the EPA-accepted Thermo Orion Method AC4P72 (using N,N-diethyl-p-phenylenediamine, thus known as DPD method; Thermo Fisher, Waltham, MA, USA) by measuring absorbance at $520 \mathrm{~nm}$ using a miniature spectrophotometer (USB4000, Ocean Optics, Inc.; Dunedin, FL, USA).

Assay Procedure. Norovirus suspensions $(5 \mu \mathrm{L})$ from spiked environmental water samples were pipetted directly to the center of each $\mu \mathrm{PAD}$ channel made out of nitrocellulose paper, without using any pre-treatments. This norovirus suspension spread through each microfluidic channel, where norovirus particles were captured onto nitrocellulose paper (polarity filter) via electrostatic interactions. After loading norovirus, $2 \mu \mathrm{L}$ of anti-norovirus-conjugated fluorescent polystyrene particle suspension $(0.001 \% \mathrm{w} / \mathrm{v}$ for DI water and $0.002 \% \mathrm{w} / \mathrm{v}$ tap water and reclaimed wastewater) was loaded onto the center of each channel on the $\mu \mathrm{PAD}$ where noroviruses were captured (Figure 1a). Anti-norovirus conjugated particles flowed through and filled the entire channel by capillary action (or wicking). These particles were aggregated by antibody-antigen binding, that is, immunoagglutination, which were imaged as described in the following section.
Imaging Particle Aggregation on $\mu$ PADs Using a Benchtop Fluorescence Microscope. Particle aggregation with norovirus was imaged by taking 4 random images of each channel with a $5 \mathrm{~s}$ exposure time, initially using a benchtop fluorescence microscope (Eclipse TS 100; Nikon Corp.; Tokyo, Japan), equipped with a fluorescence filter (A.G. Heinze B-2E/C; A.G. Heinze, Inc.; Lake Forest, CA, USA) and imaging software (NIS Elements; Nikon Corp.; Tokyo, Japan). Only green channel images were used. From the processed images, the pixel counts were evaluated, which were added together for 4 different images to yield a single data point. This procedure was repeated 3-4 times, each time using a different $\mu \mathrm{PAD}$.

Imaging Particle Aggregation on $\mu$ PADs Using a Smartphone-Based Fluorescence Microscope. The smartphone-based fluorescence microscope (Figure 1b) consisted of an external microscope (XFox Professional 300X Optical Glass Lenses; X\&Y Ind., Shenzhen, China) with magnification $200 \times$ to $300 \times$, attached to a smartphone (iPhone 7; Apple, Inc.; Cupertino, CA, USA). A blue excitation light source was provided by a secondary smartphone flashlight with a $480 \pm 10 \mathrm{~nm}$ bandpass filter (catalog number 43-115; Edmund Optics, Barrington, NJ, USA). This can be easily replaced by any blue light-emitting diode (LED). An unmounted $525 \pm 20 \mathrm{~nm}$ bandpass filter (catalog number BP525-D25; Midwest Optical Systems, Inc.; Palatine, IL, USA) was placed in between the $\mu \mathrm{PAD}$ and the objective lens of a microscope to capture green fluorescence emission. All images were taken using the ProCam 4 app (Samer Azzam, http://www.procamapp.com; downloaded via iTunes), where the exposure time and white balance could be manually adjusted. Light trail exposure time was $4 \mathrm{~s}$, white balance was 4000 , and ISO was 200. Similar to benchtop fluorescence microscopy, four images were taken from each channel to yield a single data point. Experiments were repeated 3-6 times, each time using a different $\mu \mathrm{PAD}$. 
Image Analysis for Benchtop Fluorescence Microscopic Images. ImageJ (U.S. National Institutes of Health; Bethesda, MD, USA) was initially processed on a separate desktop computer to analyze the images taken on a benchtop fluorescence microscope. For benchtop fluorescence microscopic images, "Find Edges" option in ImageJ was utilized to outline the image of particles. All pixels with intensity values $<100$ (out of 255 for green emission) were considered background noise and eliminated. This threshold value (100) was determined by comparing the images with those measured by a higher magnification fluorescence microscope. All other pixels with intensity values $\geq 100$ were selected, the interior of the edges was filled, and these selected pixels were binarized. This procedure resulted in binary images of the particles. Once the images were binarized, "Analyze Particles" function was selected in ImageJ, and the pixel area was obtained. The pixel area $<50$ was eliminated because they were single particles that were not aggregated by norovirus. This threshold value (50) was determined by comparing the images to those measured by a higher magnification fluorescence microscope. The final data consisted of the following: (1) the number of aggregated particle clusters and (2) the total accumulated pixel counts of all aggregated particles, for the given image. This procedure is schematically illustrated in Figure 7.

Image Analysis for Smartphone-Based Fluorescence Microscopic Images. All smartphone-based fluorescence microscopic images were split into red, green, and blue channels. While the maximum emission wavelength of the fluorescent particles was $525 \mathrm{~nm}$, their emission is actually ranged over $550 \mathrm{~nm}$, that is, boundary of green and red colors (hence, they are referred to as "yellow-green" particles). Therefore, their fluorescence emission could be captured in not only green but also red channels. Because nitrocellulose paper absorbed and scattered light at most wavelengths (its color is bright white) and the maximum exposure time of a smartphone camera was much shorter than that of a benchtop fluorescence microscope, the pixel intensities were quite low. Therefore, both green and red channels were combined to maximize the pixel intensities. Unlike the benchtop fluorescence microscopy, the mean pixel intensities of combined green and red channel images were evaluated using an original code developed in MATLAB version R2017a (The MathWorks, Inc.; Natick, MA, USA). A GUI (Figure S1) was created and used to automate the analysis procedure and to provide its user-friendliness.

Smartphone microscopic images were processed using a similar algorithm to the benchtop fluorescence microscopy and ImageJ processing. Because the bright-field views of smartphone microscopic images were circular in shape, all images were cropped into squares circumscribing those circles, such that all pixels could be utilized for analyses. Aggregated fluorescent particles always exhibited the combined green and red pixel intensities substantially higher than the overall mean intensities of the cropped area. To eliminate background noise and isolate only the particles, cut-off intensities were applied to the images set at overall mean intensity +40 to 50 . The resulting images were then binarized. To eliminate the nonaggregated particles, those with a pixel area $<30$ were eliminated from the binarized images. This cut-off value of a 30 pixel area was smaller than that of benchtop fluorescence microscopy, 50 because of the lower magnification and narrower dynamic range of smartphone-acquired images. This threshold filtering successfully eliminated all ambient light variations, indicating that the method is appropriate for field use. Again, this procedure is schematically illustrated in Figure 7. The MATLAB GUI generated the accumulated pixel counts of all aggregated particles, for the given image. The MATLAB code and its GUI were adapted to be executed within MATLAB mobile (The MathWorks, Inc.; Natick, MA, USA) to enable the image analysis performed within a smartphone (Figure S2). Once images were acquired, the total assay time was less than $1 \mathrm{~min}$ including the time for user input.

Statistical Analysis. Four different images were taken from each $\mu \mathrm{PAD}$ channel (Figure 1A) and the sum of pixel counts from these four images (representing the extent of particle aggregation) was recorded for the given concentration of norovirus. These experiments were repeated 3-6 times for each concentration of norovirus, each time using different $\mu \mathrm{PAD}$. Averages of these 3-6 $\mu \mathrm{PAD}$ assays were recorded. $P$ values for each norovirus concentration against the negative control sample (unspiked) were calculated using the Wilcoxon rank sum test, performed with JMP software version 14.3.0 (SAS Institute, Inc.; Cary, NC, USA) with $\alpha=0.05$.

\section{ASSOCIATED CONTENT}

\section{S Supporting Information}

The Supporting Information is available free of charge on the ACS Publications website at DOI: 10.1021/acsomega.9b00772.

Representative raw (left), background-removed (middle), and non-aggregated particles-removed images (right) of a $\mu \mathrm{PAD}$, captured by a benchtop fluorescence (top) and light (bottom) microscope and processed with ImageJ (Figure S1); User interface of an original, MATLAB GUI app (Figure S2); Processed $\mu \mathrm{PAD}$ channel images using a MATLAB code from smartphone fluorescence microscopic images (Figure S3); Smartphone assay results for $0.5 \mathrm{ppm}$ (left) and $5 \mathrm{ppm}$ (right) chlorine added to DI water (Figure S4) (PDF)

\section{AUTHOR INFORMATION}

\section{Corresponding Author}

*E-mail: jyyoon@email.arizona.edu.

\section{ORCID}

Jeong-Yeol Yoon: 0000-0002-9720-6472

\section{Author Contributions}

J.-Y.Y. conceived the overall concept with input from S.C. and K.A.R. S.C. designed, assembled and fabricated $\mu$ PADs and the smartphone based fluorescence microscope, and conducted all benchtop and smartphone microscope assay experiments, with assistance from L.E.B. and S.P. S.C. and J.-Y.Y. developed an image processing procedure. S.C. analyzed all data, with assistance from L.E.B. and input from J.-Y.Y. and K.A.R. W.Q.B. and C.M.M. collected water samples from the water reclamation facility and analyzed them by RT-qPCR. J.-Y.Y. and S.C. wrote the manuscript with input from L.E.B., S.P., W.Q.B. and K.A.R. All authors have given approval to the final version of the manuscript.

\section{Funding}

Funding for this research was provided by the University of Arizona National Science Foundation Water and Environmental Technology (WET) Center (award number IIP1361815) and Tucson Water. 


\section{Notes}

The authors declare no competing financial interest.

\section{ACKNOWLEDGMENTS}

The authors are grateful to Dr. Ian L. Pepper and Dr. Charles P. Gerba, both at Department of Soil, Water and Environmental Science and Water and Energy Sustainable Technology (WEST) Center at the University of Arizona, as well as Daniel R. Quintanar at Tucson Water, for helpful discussions and suggestions for this project.

\section{REFERENCES}

(1) Katayama, H.; Vinjé, J. Norovirus and other Calicivirus. Global Water Pathogens Project: Michigan State University, UNESCO: East Lansing, 2017. http://www.waterpathogens.org/book/norovirus-andother-caliciviruses.

(2) Teunis, P. F. M.; Moe, C. L.; Liu, P.; Miller, S. E.; Lindesmith, L.; Baric, R. S.; Le Pendu, J.; Calderon, R. L. Norwalk virus: how infectious is it? J. Med. Virol. 2008, 80, 1468-1476.

(3) Atmar, R. L.; Opekun, A. R.; Gilger, M. A.; Estes, M. K.; Crawford, S. E.; Neill, F. H.; Ramani, S.; Hill, H.; Ferreira, J.; Graham, D. Y. Determination of the $50 \%$ human infectious dose for Norwalk virus. J. Infect. Dis. 2014, 209, 1016-1022.

(4) Ettayebi, K.; Crawford, S. E.; Murakami, K.; Broughman, J. R.; Karandikar, U.; Tenge, V. R.; Neill, F. H.; Blutt, S. E.; Zeng, X.-L.; Qu, L.; Kou, B.; Opekun, A. R.; Burrin, D.; Graham, D. Y.; Ramani, S.; Atmar, R. L.; Estes, M. K. Replication of human noroviruses in stem cell-derived human enteroids. Science 2016, 353, 1387-1393.

(5) Coudray-Meunier, C.; Fraisse, A.; Martin-Latil, S.; Guillier, L.; Delannoy, S.; Fach, P.; Perelle, S. A comparative study of digital RTPCR and RT-qPCR for quantification of Hepatitis A virus and Norovirus in lettuce and water samples. Int. J. Food Microbiol. 2015, 201, 17-26.

(6) de Bruin, E.; Duizer, E.; Vennema, H.; Koopmans, M. P. G. Diagnosis of Norovirus outbreaks by commercial ELISA or RT-PCR. J. Virol. Methods 2006, 137, 259-264.

(7) Park, J. P.; Cropek, D. M.; Banta, S. High affinity peptides for the recognition of the heart disease biomarker troponin I identified using phage display. Biotechnol. Bioeng. 2010, 105, 678-86.

(8) $\mathrm{Mu}$, X.; Zhang, L.; Chang, S.; Cui, W.; Zheng, Z. Multiplex microfluidic paper-based immunoassay for the diagnosis of hepatitis $\mathrm{C}$ virus infection. Anal. Chem. 2014, 86, 5338-5344.

(9) Hu, J.; Wang, S.; Wang, L.; Li, F.; Pingguan-Murphy, B.; Lu, T. J.; Xu, F. Advances in paper-based point-of-care diagnostics. Biosens. Bioelectron. 2014, 54, 585-597.

(10) Du, Y.; Pothukuchy, A.; Gollihar, J. D.; Nourani, A.; Li, B.; Ellington, A. D. Coupling sensitive nucleic acid amplification with commercial pregnancy test strips. Angew. Chem., Int. Ed. 2017, 56, 992-996.

(11) Meng, X.-Y.; Gao, Y.; Zhang, H.; Luo, Y.; Sun, Y.; Qiu, H.-J. Cross-priming amplification-based lateral flow strip as a novel tool for rapid on-site detection of wild-type Pseudorabies virus. Sens. Actuators, B 2018, 259, 573-579.

(12) Rohrman, B. A.; Richards-Kortum, R. A Paper and Plastic Device for Performing recombinase polymerase amplification of HIV DNA. Lab Chip 2012, 12, 3082-3088.

(13) Greening, G. E.; Cannon, J. L. Human and animal viruses in food (including taxonomy of enteric viruses). Viruses in Foods; Springer: Berlin, 2016.

(14) Weng, X.; Neethirajan, S. Aptamer-based fluorometric determination of norovirus using a paper-based microfluidic device. Microchim. Acta 2017, 184, 4545-4552.

(15) Hagström, A. E. V.; Garvey, G.; Paterson, A. S.; Dhamane, S.; Adhikari, M.; Estes, M. K.; Strych, U.; Kourentzi, K.; Atmar, R. L.; Willson, R. C. Sensitive detection of norovirus using phage nanoparticle reporters in lateral-flow assay. PLoS One 2015, 10, No. e0126571.
(16) Han, K. N.; Choi, J.-S.; Kwon, J. Three-dimensional paperbased slip device for one-step point-of-care testing. Sci. Rep. 2016, 6, 25710.

(17) Cho, S.; Park, T. S.; Nahapetian, T. G.; Yoon, J.-Y. Smartphone-based, sensitive $\mu \mathrm{PAD}$ detection of urinary tract infection and gonorrhea. Biosens. Bioelectron. 2015, 74, 601-611.

(18) Fujita, M.; Adachi, K.; Nagasawa, M. Development of a homogeneous time-resolved fluorescence assay for detection of viral double-stranded RNA. Anal. Biochem. 2019, 566, 46-49.

(19) Costantini, V.; Morantz, E. K.; Browne, H.; Ettayebi, K.; Zeng, X.-L.; Atmar, R. L.; Estes, M. K.; Vinjé, J. Human norovirus replication in human intestinal enteroids as model to evaluate virus inactivation. Emerg. Infect. Dis. 2018, 24, 1453-1464.

(20) Park, T. S.; Yoon, J.-Y. Smartphone detection of Escherichia coli from field water samples on paper microfluidics. IEEE Sens. J. 2015, $15,1902-1907$.

(21) Betancourt, W. Q.; Kitajima, M.; Wing, A. D.; Regnery, J.; Drewes, J. E.; Pepper, I. L.; Gerba, C. P. Assessment of virus removal by managed aquifer recharge at three full-scale operations. J. Environ. Sci. Health, Part A: Environ. Sci. Eng. 2014, 49, 1685-1692.

(22) Kageyama, T.; Kojima, S.; Shinohara, M.; Uchida, K.; Fukushi, S.; Hoshino, F. B.; Takeda, N.; Katayama, K. Broadly reactive and highly sensitive assay for Norwalk-like viruses based on real-time quantitative reverse transcription-PCR. J. Clin. Microbiol. 2003, 41, $1548-1557$.

(23) Kitajima, M.; Oka, T.; Haramoto, E.; Takeda, N.; Katayama, K.; Katayama, H. Seasonal distribution and genetic diversity of genogroups I, II, and IV noroviruses in the Tamagawa River, Japan. Environ. Sci. Technol. 2010, 44, 7116-7122.

(24) Ahmed, S. R.; Takemeura, K.; Li, T.-C.; Kitamoto, N.; Tanaka, T.; Suzuki, T.; Park, E. Y. Size-controlled preparation of peroxidaselike graphene-gold nanoparticle hybrids for the visible detection of norovirus-like particles. Biosens. Bioelectron. 2017, 87, 558-565.

(25) Ashiba, H.; Sugiyama, Y.; Wang, X.; Shirato, H.; HigoMoriguchi, K.; Taniguchi, K.; Ohki, Y.; Fujimaki, M. Detection of norovirus virus-like particles using a surface plasmon resonanceassisted fluoroimmunosensor optimized for quantum dot fluorescent labels. Biosens. Bioelectron. 2017, 93, 260-266. 